\title{
Dynamical Multistability Induced by Radiation Pressure in High-Finesse Micromechanical Optical Cavities
}

\author{
Florian Marquardt, ${ }^{1,2}$ J. G. E. Harris, ${ }^{2}$ and S. M. Girvin ${ }^{2}$ \\ ${ }^{1}$ Physics Department, Center for NanoScience, and Arnold Sommerfeld Center for Theoretical Physics, \\ Ludwig-Maximilians University Munich, Theresienstrasse 37, 80333 Munich, Germany \\ ${ }^{2}$ Departments of Physics and Applied Physics, Yale University, P.O. Box 208284, New Haven, Connecticut 06520, USA
}

(Received 24 February 2005; published 16 March 2006)

\begin{abstract}
We analyze the nonlinear dynamics of a high-finesse optical cavity in which one mirror is mounted on a flexible mechanical element. We find that this system is governed by an array of dynamical attractors, which arise from phase locking between the mechanical oscillations of the mirror and the ringing of the light intensity in the cavity. We develop an analytical theory to map out the diagram of attractors in parameter space, derive the slow amplitude dynamics of the system, including thermal fluctuations, and suggest a scheme for exploiting the dynamical multistability in the measurement of small displacements.
\end{abstract}

Introduction. - The radiation pressure exerted by light stored in an optical cavity couples the cavity mirrors' mechanical degrees of freedom to the optical field. The resulting dynamics offer novel means for manipulating both the light and the mirrors, for example, by generating squeezed [1,2] or entangled states of light [3] or matter [4], or by tailoring the mechanical properties of the mirrors [510]. The radiation pressure also sets limits on the sensitivity of a range of experiments, e.g., by enforcing the standard quantum limit for displacement measurements [1115] or introducing mechanical instabilities [2,16-18].

To date the theoretical description of these systems has been mostly developed for small mirror oscillations. As advances in fabrication [19-24] produce devices with stronger optomechanical coupling and weaker damping, nonlinearities become increasingly important. This has been illustrated by recent micromechanical experiments that have observed the dynamical instability $[9,25,26]$ which is the starting point for our analysis. Here we develop a description of this regime that is equally applicable to the Caltech whispering-gallery cavity experiment and a setup currently being prepared at Yale $[25,26]$. In both, the rescaled radiation pressure $(\mathcal{P}$, defined below) can be more than 15 orders of magnitude larger than in previous experiments. For setups operating in this novel regime, we predict a new kind of "dynamical multistability," where the system develops an array of stable dynamical attractors. We interpret this as phase locking between the mirror motion and the ringing in the cavity optical field. We develop an analytical theory that is much more efficient than numerical simulations of the microscopic equations of motion for the parameter regime of interest here, and which includes the effects of fluctuations.

The model. -We consider the setup of Fig. 1. The Hamiltonian of a given cavity mode is $\hat{H}_{\text {cav }}=\hbar \omega_{L}(1-$ $\left.\hat{x}_{\mathrm{CL}} / l\right) \hat{a}^{\dagger} \hat{a}$. The cavity of length $l$ is resonant with the laser frequency $\omega_{L}$ when the cantilever (CL) position $x_{\mathrm{CL}}$ is zero, and we assume the excursions in $x_{\mathrm{CL}}$ to be small enough to avoid the other optical resonances (we also neglect the finite travel time of the light between the mirrors). The radiation pressure force is $\hat{F}=\left(\hbar \omega_{L} / l\right) \hat{a}^{\dagger} \hat{a}$. In the following we will consider the purely classical nonlinear dynamics, where $\hat{a}(t)$ is replaced by the coherent light amplitude $\tilde{\alpha}(t)$. We use the ring-down time of the cavity $\gamma^{-1}$, the resonance width $\delta x=l \gamma / \omega_{L}$, and the cantilever mass $m$ as convenient new units, and rescale $\alpha$ such that it becomes 1 at resonance: $\alpha=\tilde{\alpha} e^{i \omega_{L} t} / \sqrt{n_{\max }}$, where the maximum photon number $n_{\max }=4 P_{\text {in }} /\left(\gamma \hbar \omega_{L}\right)$ is linear in the input power $P_{\text {in }}$. Then the coupled equations of motion for $\alpha$ and $x=x_{\mathrm{CL}} / \delta x$ read [2]

$$
\begin{gathered}
\dot{\alpha}=\left(i x-\frac{1}{2}\right) \alpha+\frac{1}{2}, \\
\ddot{x}=\mathcal{P}|\alpha|^{2}-\omega_{0}^{2}\left(x-x_{0}\right)-\Gamma \dot{x} .
\end{gathered}
$$

Additional nonlinear terms in Eq. (2) (e.g., due to strain nonlinearity) will not play any role for the amplitudes considered here. The oscillator frequency $\omega_{0}$ and mechanical damping rate $\Gamma$ are fixed, while the detuning from resonance $x_{0}$ may be controlled, either by changing the laser frequency or applying a static force to the cantilever. All the other constants are combined into the dimensionless input power $\mathcal{P}=4 P_{\text {in }} \omega_{L} / m \gamma^{4} l^{2}=\hbar n_{\max } / m \delta x^{2} \gamma$. We emphasize that the scale of the radiation pressure, set by $\mathcal{P}$, grows with the fourth power of the cavity finesse [ $\mathcal{P} \propto \gamma^{-4}$ with $\gamma=(c / 2 l) \mathcal{T}$, where $\mathcal{T}$ is the mirror transmission]. The nonlinear effects we investigate become important for $\Gamma \ll \mathcal{P}$.

The fabrication process described in [21,22] may be used to integrate cantilevers with high-reflectivity dielec-

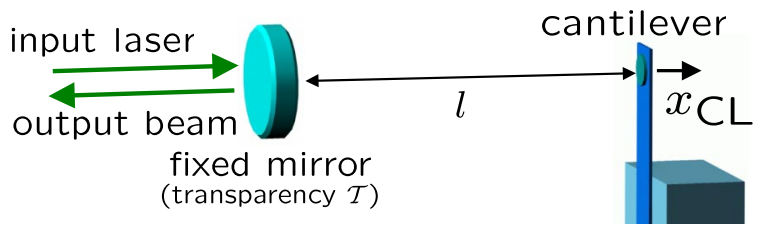

FIG. 1 (color online). The setup analyzed in the text. 
tric mirrors as part of a high-finesse optical cavity at low temperatures. With modest adjustments to the dimensions of [22], it should be possible to realize cantilevers with spring constant $\sim 1 \mathrm{~N} / \mathrm{m}$, mass $m \sim 10^{-10} \mathrm{~kg}$, and mechanical $Q \sim 10^{5}$ supporting a mirror capable of achieving a cavity finesse of $\pi / \mathcal{T} \sim 10^{5}$ (with $l \sim 10 \mathrm{~cm}$ ), and use them at an input power of $P_{\text {in }} \sim 20 \mathrm{nW}$ at $T \sim 0.3 \mathrm{~K}$. For the Yale (Caltech $[25,26])$ setups, our dimensionless units are scaled by $\delta x \approx 3 \mathrm{pm}(3.5 \mathrm{pm}), \delta t=\gamma^{-1} \approx 10 \mu \mathrm{s}$ (19 ns), and we have $\Gamma \approx 10^{-5}\left(5.4 \times 10^{-4}\right)$ and $\omega_{0} \approx$ $1(0.65)$. For $P_{\text {in }} \approx 20 \mathrm{nW}(5 \mathrm{~mW})$, we find $\mathcal{P} \approx 1(9.5)$, and $|\alpha|^{2}=1$ corresponds to an energy of $0.8 \mathrm{pJ}(0.4 \mathrm{~nJ})$ or $2 \times 10^{6}\left(3 \times 10^{9}\right)$ photons inside the cavity. An energy $U=1$ or $\tilde{T}=1$ (see below) equals $0.6 \mathrm{~K}\left(10^{5} \mathrm{~K}\right)$. Note that in the Munich experiment [9] the optical resonances overlap and $\mathcal{P} \sim 10^{-18} \ll \Gamma$ precludes the multistability.

The cavity resonance peak $\alpha(x)=1 /(1-2 i x)$ gives rise to a barrier in the effective static cantilever potential obtained by integrating the right-hand-side (rhs) of Eq. (2), $V_{\text {eff }}(x)=\frac{\omega_{0}^{2}}{2}\left(x-x_{0}\right)^{2}-\frac{\mathcal{P}}{2} \arctan (2 x)$. There can be two local minima of $V_{\text {eff }}$, leading to static bistability [6,7]. However, it is known [5] that the time lag generated by the finite cavity ring-down time $\gamma^{-1}$ introduces additional damping or antidamping when $x$ is to the left or right of the barrier, respectively. Here we will focus on the regime where the antidamping leads to an instability discovered previously $[2,16,17,27]$, which destroys the stable solution $\dot{x}=0$. Then the system settles into self-sustained oscillations, whose full nonlinear dynamics we explore here.

Dynamics in the unstable regime.-For the parameters from above, the effects of radiation during one cycle are weak, such that $x(t)$ carries out approximately sinusoidal oscillations at the unperturbed frequency: $x(t) \approx \bar{x}+$ $A \cos \left(\omega_{0} t\right)$. This fact is the basis of our analytical theory. For very high $\mathcal{P}$ this approximation breaks down and chaotic motion may result (as was observed in [25] for $\mathcal{P} \sim 6000$ ), which will not be analyzed here. The dynamics of the light amplitude $\alpha(t)$ resembles that of a driven damped oscillator which is swept through resonance nonadiabatically (see Fig. 2). The exact solution for a given sinusoidal $x(t)$ can be written as a Fourier series, $\alpha(t)=$ $e^{i \varphi(t)} \sum_{n} \alpha_{n} e^{i n \omega_{0} t}$, with

$$
\alpha_{n}=\frac{1}{2} \frac{J_{n}\left(-\frac{A}{\omega_{0}}\right)}{i n \omega_{0}+\frac{1}{2}-i \bar{x}},
$$

and a global phase $\varphi(t)=\left(A / \omega_{0}\right) \sin \left(\omega_{0} t\right)$, where $J_{n}$ is the Bessel function of the first kind. The output light intensity is [28] given by $P_{\text {in }}|1-2 \alpha|^{2}$, and the Fourier transform of $\alpha(t)$ directly yields the sideband spectrum.

Dynamical multistability. - The possible attractors $(\bar{x}, A)$ have to fulfill two conditions resulting from Eq. (2) for any periodic motion. The total time-averaged force $\langle\ddot{x}\rangle$ has to vanish, and the net power input via the radiation pressure force (due to the Doppler shift caused by the moving mirror) must equal the power dissipated through friction, $\langle\ddot{x} \dot{x}\rangle=0$ :
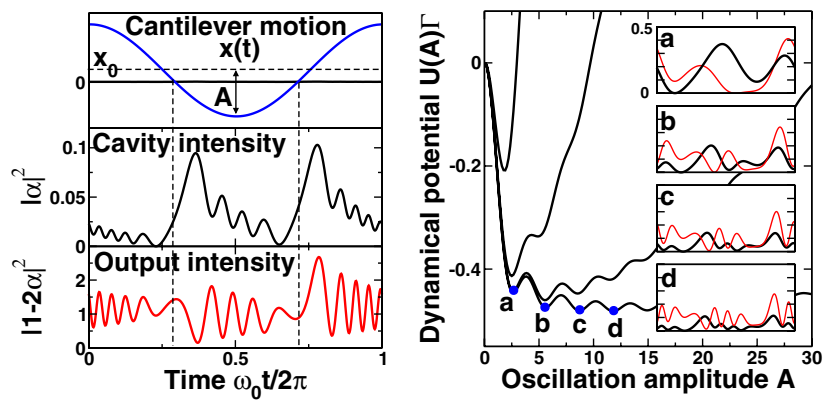

FIG. 2 (color online). Left: Oscillations of the light energy stored inside the cavity, $\hbar \omega_{L} n_{\max }|\alpha|^{2}$, and the output light intensity $P_{\text {out }}=P_{\text {in }}|1-2 \alpha|^{2}$, for given sinusoidal cantilever motion (with $\omega_{0}=1, \bar{x}=x_{0}=5, A=20$ ). See text for real units. Right: Dynamical potential $U(A)$, for $\mathcal{P}=1$ and $\Gamma=$ $10^{-4}, 10^{-3}, 10^{-2}, 10^{-1}$ (bottom to top curve). Insets show the time evolution of the cavity intensity $|\alpha|^{2}$ (thick line) and the output intensity $|1-2 \alpha|^{2}$ (thin line, scaled down by a factor 0.1 ), obtained for oscillations corresponding to the dynamical attractors a,b,c,d [minima in $U(A)$ ]. Note the additional oscillation in $|\alpha|^{2}$ for each subsequent attractor.

$$
\begin{gathered}
\mathcal{P}\left\langle|\alpha(t)|^{2}\right\rangle=\omega_{0}^{2}\left(\bar{x}-x_{0}\right), \\
P_{\text {rad }}=\mathcal{P}\left\langle|\alpha(t)|^{2} \dot{x}\right\rangle=P_{\text {fric }}=\Gamma\left\langle\dot{x}^{2}\right\rangle .
\end{gathered}
$$

The dependence on $\bar{x}, A$, and $\omega_{0}$ follows from the coefficients of Eq. (3):

$$
\begin{gathered}
\left\langle|\alpha|^{2}\right\rangle=\sum_{n}\left|\alpha_{n}\right|^{2}, \\
\tilde{P}_{\mathrm{rad}}=\left\langle|\alpha|^{2} \dot{x}\right\rangle=A \omega_{0} \operatorname{Im} \sum_{n} \alpha_{n}^{*} \alpha_{n+1} .
\end{gathered}
$$

We note that a force following the light intensity with a time lag $\tau$ (e.g., photothermal forces [9]) would enter Eq. (2) in the form $\mathcal{P} \tau^{-1} \int_{-\infty}^{t} d t^{\prime}|\alpha|^{2}\left(t^{\prime}\right) \exp [-(t-$ $\left.\left.t^{\prime}\right) / \tau\right]$, and leads to a factor $\left(1+i \omega_{0} \tau\right)^{-1}$, inside the imaginary part on the rhs of Eq. (7).

The power balance equation can be recast into the form

$$
\frac{\tilde{P}_{\text {rad }}(\bar{x}, A)}{\tilde{P}_{\text {fric }}(A)}=\frac{\Gamma}{\mathcal{P}},
$$

with $\tilde{P}_{\text {fric }}(A)=\omega_{0}^{2} A^{2} / 2$. Stable attractors are those where the ratio decreases for increasing $A$.

After solving the force balance Eq. (4) for $\bar{x}=\bar{x}\left(x_{0}, A\right)$, we can plot the contour lines of the left-hand side of Eq. (8) in the $\left(x_{0}, A\right)$ plane. These yield the possible equilibrium values of the oscillation amplitude $A$ as a function of $x_{0}$, see Fig. 3. The red dots show the results of a simulation of the initial equations of motion, Eqs. (1) and (2).

This diagram may be observed experimentally by sweeping $x_{0}$, either via tuning the laser frequency or by applying some force to the cantilever. After initial transients, the system settles into one of the attractors (specific amplitude $A$ ), which may be identified either via its distinct pattern of light emission from the cavity (see Fig. 2) or by the total power loss due to friction, $P_{\text {in }}-\bar{P}_{\text {out }}=$ $P_{\text {in }} \Gamma A^{2} \omega_{0}^{2} / 2$, or by obtaining $x(t)$ from interferometry 


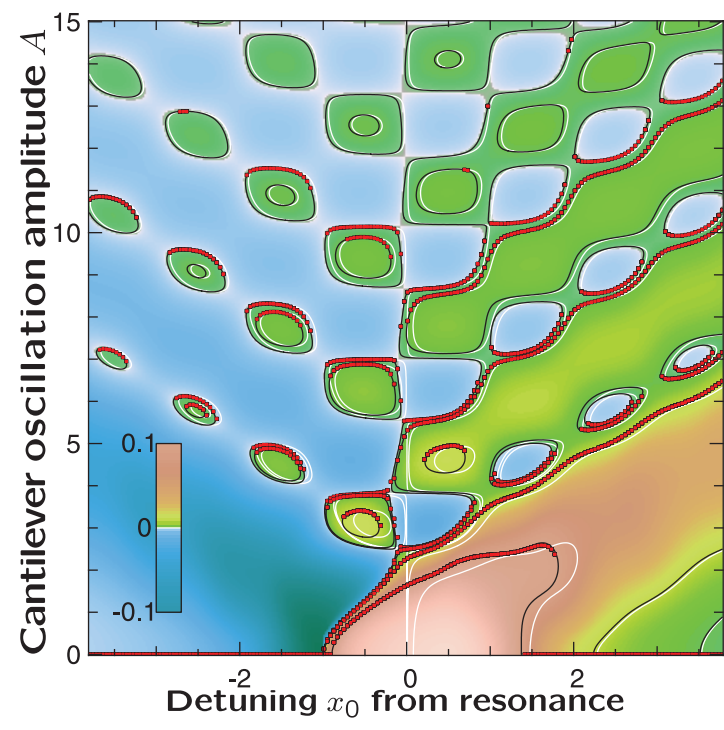

FIG. 3 (color). Density plot of the ratio of reduced radiation power input and frictional power loss, $\tilde{P}_{\text {rad }}\left(\bar{x}\left(x_{0}, A\right), A\right) / \tilde{P}_{\text {fric }}(A)$, in the $\left(x_{0}, A\right)$ plane. The contour lines indicate possible cantilever oscillation amplitudes $A$, according to Eq. (8). White contour lines display the approximation $\bar{x} \approx x_{0}$. Contours are drawn for $\Gamma / \mathcal{P}=10^{-4}, 10^{-3}, 10^{-2}, 10^{-1}$ (with $\mathcal{P}=\omega_{0}=1$ ). Red dots show the long-time limit $\left(t_{\text {sim }}=2 \times 10^{4}\right)$ of the amplitude $A=$ $x_{\max }-x_{\min }$ in the numerically exact solution of the original equations of motion, obtained for the same values of $\Gamma / \mathcal{P}$ and a set of random initial conditions.

with a detuned beam. Sweeping back nonadiabatically to smaller $x_{0}$ allows one to reach attractors with higher $A$, even those that persist at negative $x_{0}$, where the system would not start oscillating spontaneously.

Near the onset of instability at $x_{0}^{*}=-\mathcal{P} / \omega_{0}^{2}$ we find $A \approx\left[\left(1+4 \omega_{0}^{2}\right)\left(1+\omega_{0}^{2} x_{0} / \mathcal{P}\right) / 2\right]^{1 / 2}$ (for $\Gamma \ll \mathcal{P}$ ), and $|\bar{x}| \ll x_{0}$. However, for the rest of parameter space, $\bar{x} \approx$ $x_{0}$ is a good approximation (white lines in Fig. 3), which we will adopt in the following. Then the equilibrium values of $A$, at a given $x_{0}$, depend only on the ratio $\Gamma / \mathcal{P}$.

At large $A / \omega_{0}$, the asymptotics of the Bessel function in Eq. (3) leads to an oscillatory decay of $\tilde{P}_{\text {rad }} / \tilde{P}_{\text {fric }}$ (see Fig. 3), with periods of $\pi \omega_{0}$ and $2 \omega_{0}$ in $A$ and $x_{0}$, respectively. Physically, an increase in $A$ enlarges the timedependent detuning $x(t)$ which is equal to the frequency of the ringing in the light intensity $|\alpha|^{2}(t)$ (Fig. 2). The appearance of the array of attractors is thus due to a phase locking phenomenon, where the oscillations of the light intensity (depending on $A$ ) seek to be commensurate with the fundamental cantilever period.

Amplitude equation of motion. - We can determine the slow dynamics of the cantilever oscillation amplitude $A$ by equating the change in total energy $E=\left(\omega_{0}^{2} / 2\right) A^{2}$ to the net power input:

$$
\frac{d E}{d t}=P_{\text {total }}=P_{\text {rad }}-P_{\text {fric }}
$$

This can be used to obtain the overdamped motion of $A$,

$$
\frac{d A}{d t}=\frac{1}{A \omega_{0}^{2}} P_{\text {total }}=-\frac{\Gamma}{2 \omega_{0}^{2}} U^{\prime}(A),
$$

where we have introduced the effective potential for $A$,

$$
U(A)=\frac{\omega_{0}^{2}}{2} A^{2}-\frac{2 \mathcal{P}}{\Gamma} \int_{A_{0}}^{A} \tilde{P}_{\mathrm{rad}}\left(A^{\prime}\right) \frac{d A^{\prime}}{A^{\prime}} .
$$

The integral produces a decaying oscillating component, such that $U(A)$ in general displays several local minima, corresponding to the dynamical attractors (see Fig. 2, right). Solving Eq. (10) and comparing to simulations of the full dynamics yields a good agreement, which can be improved at small $\left|x_{0}\right|$ by using $\bar{x}=\bar{x}\left(x_{0}, A\right)$ from Eq. (4) instead of $\bar{x}=x_{0}$. This was also used for Fig. 4.

Stochastic dynamics and Boltzmann distribution.-The heat bath responsible for the mechanical damping $\Gamma$ also produces a fluctuating force, which enters the rhs of Eq. (2) as $\xi_{x}(t)$, where $\left\langle\xi_{x}(t) \xi_{x}(0)\right\rangle=2 \Gamma \tilde{T} \delta(t)$, with a reduced temperature $\tilde{T}=k_{B} T /\left(m \delta x^{2} \gamma^{2}\right)$. This gives rise to a fluctuating power $\xi_{E}=\dot{x} \xi_{x}$ in Eq. (9), which produces a stochastic force $\xi_{A}(t)=\xi_{E}(t) /\left(A \omega_{0}^{2}\right)$ on the rhs of (10), with $\left\langle\xi_{A}(t) \xi_{A}(0)\right\rangle=\left(\Gamma \tilde{T} / \omega_{0}^{2}\right) \delta(t)$. The cantilever settles into a driven equilibrium described by the Boltzmann distribution $w(A) \propto A \exp (-U(A) / \tilde{T})$ (Fig. 4). The prefactor $A$ accounts for the phase space associated with the two quadratures of motion. Formally, it is produced by an additional deterministic term $\Gamma \tilde{T} /\left(2 \omega_{0}^{2} A\right)$ on the rhs of Eq. (10), which stems from rewriting the Langevin version of (9) in Ito form. Comparison with a Langevin-RungeKutta [29] simulation of the original Langevin equations for $x$ and $\alpha$ shows good agreement (shot noise may be neglected as $n_{\max } \sim 10^{6} \gg 1$ ). At $\left|x_{0}\right| \gg 1$, the global minimum of $U(A)$ is at $A=0$, and $w(A)$ returns to the distribution of the unperturbed oscillator.

The potential barriers in $U(A)$ scale with $\mathcal{P} / \Gamma$, see Eq. (11), and can become much larger than those in $V_{\text {eff }}(x)$, which scale with $\mathcal{P}$. For the Yale (Caltech) setups,

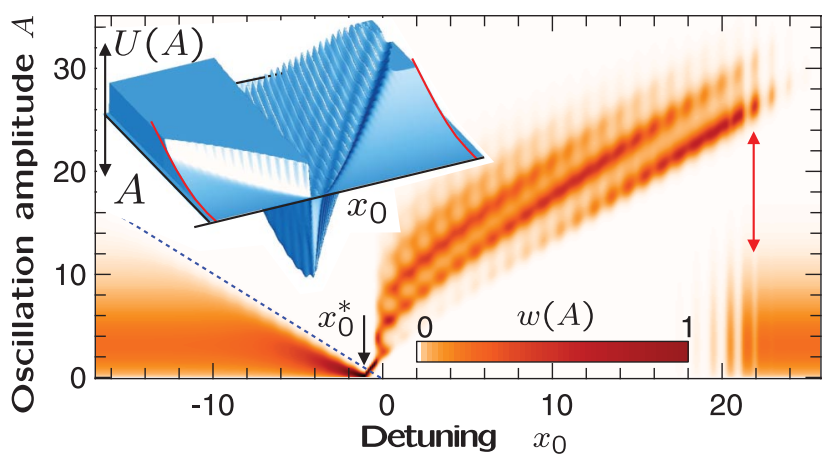

FIG. 4 (color). Boltzmann distribution $w(A)$ of the cantilever oscillation amplitude $A$, as a function of detuning $x_{0}$, for a reduced temperature $\tilde{T}=10$. Other parameters: $\mathcal{P}=\omega_{0}=1$, $\Gamma=10^{-3}$. Note the narrowing (cooling) of the distribution for $x_{0}<0$ (dashed blue line), the onset of instability at $x_{0}^{*}$, and the transition(s) above $x_{0}=20$, back to the distribution of a free oscillator. Inset: Effective potential $U(A)$ (top truncated), with bare oscillator potential $\omega_{0}^{2} A^{2} / 2$ indicated in red. 

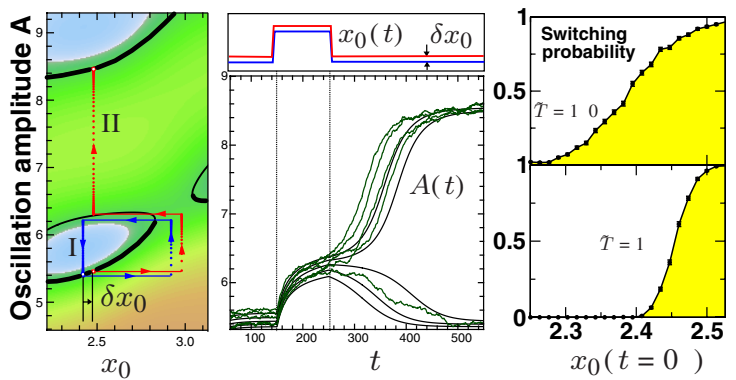

FIG. 5 (color online). Left: Two trajectories $\left(x_{0}(t), A(t)\right)$ in a latching measurement, reaching a different attractor depending on a small initial displacement $\delta x_{0}$ (parameters as in Fig. 4). Middle: Time evolution $A(t)$ for several different values of $x_{0}(t=0)$ (with/without thermal fluctuations). Right: Probability to end up in the upper attractor, vs $x_{0}(t=0)$, for two different temperatures. A sharper rise enables a more precise measurement of $\delta x_{0}$.

they are roughly $10^{3} \mathrm{~K}\left(10^{7} \mathrm{~K}\right)$, making thermal effects practically unobservable. However, a value of $\Gamma \sim 10^{-3}$ could yield barriers around $10 \mathrm{~K}$.

Hysteresis and "latching" measurements. -Multistability leads to hysteresis during parameter sweeps. Furthermore, the sensitivity to parameter perturbations is greatly enhanced near transitions between attractors. This could be used to measure small displacements $\delta x_{0}$ : Sweeping back and forth in $x_{0}$ leaves the cantilever in either of two attractors, depending on whether $\delta x_{0}$ was large enough. Afterwards, the stable final amplitude is measured. The resolution limit due to thermal fluctuations is in the subpicometer range already for $\Gamma \sim 10^{-3}$ (see Fig. 5), and becomes a few femtometers for $\Gamma \sim 10^{-5}$ (Yale setup; similar for Caltech). A similar latching scheme has been implemented in the context of a strongly driven Josephson junction [30-33].

Conclusions. - We have discovered a form of dynamical multistability that can arise in high-finesse cavities with a large mechanical quality factor of the oscillating mirror. The amplitude of self-sustained oscillations settles into one of several attractors, which we have mapped out in parameter space. We have derived an effective dynamical potential for the slow amplitude dynamics and incorporated the effects of thermal noise. Finally, we have pointed out a latching scheme for detecting small displacements. The effects described here are measurable in cavities within the reach of current technology.

We thank J. Gambetta for useful hints on stochastic simulations. F. M. acknowledges the support of the DFG. S. M. G. acknowledges support by NSF-ITR 0325580 and NSF-DMR 0342157.

[1] L. Hilico, J. Courty, C. Fabre, E. Giacobino, I. Abram, and J. Oudar, Appl. Phys. B 55, 202 (1992).

[2] C. Fabre, M. Pinard, S. Bourzeix, A. Heidmann, E. Giacobino, and S. Reynaud, Phys. Rev. A 49, 1337 (1994).
[3] V. Giovannetti, S. Mancini, and P. Tombesi, Europhys. Lett. 54, 559 (2001).

[4] W. Marshall, C. Simon, R. Penrose, and D. Bouwmeester, Phys. Rev. Lett. 91, 130401 (2003).

[5] V. B. Braginsky and A. B. Manukin, Sov. Phys. JETP 25, 653 (1967).

[6] A. Dorsel, J.D. McCullen, P. Meystre, E. Vignes, and H. Walther, Phys. Rev. Lett. 51, 1550 (1983).

[7] P. Meystre, E. M. Wright, J. D. McCullen, and E. Vignes, J. Opt. Soc. Am. B 2, 1830 (1985).

[8] M. Vogel, C. Mooser, K. Karrai, and R. J. Warburton, Appl. Phys. Lett. 83, 1337 (2003).

[9] C. Höhberger-Metzger and K. Karrai, Nature (London) 432, 1002 (2004).

[10] B.S. Sheard, M. B. Gray, C. M. Mow-Lowry, D. E. McClelland, and S.E. Whitcomb, Phys. Rev. A 69, 051801(R) (2004).

[11] C. M. Caves, Phys. Rev. Lett. 45, 75 (1980).

[12] V. B. Braginsky, M. L. Gorodetsky, F. Y. Khalili, A. B. Matsko, K.S. Thorne, and S.P. Vyatchanin, Phys. Rev. D 67, 082001 (2003).

[13] I. Tittonen et al., Phys. Rev. A 59, 1038 (1999).

[14] J. A. Sidles et al., Rev. Mod. Phys. 67, 249 (1995).

[15] C. M. Mow-Lowry, B. S. Sheard, M. B. Gray, D. E. McClelland, and S.E. Whitcomb, Phys. Rev. Lett. 92, 161102 (2004).

[16] J. M. Aguirregabiria and L. Bel, Phys. Rev. A 36, 3768 (1987).

[17] L. Bel, J. L. Boulanger, and N. Deruelle, Phys. Rev. A 37, 1563 (1988).

[18] V. B. Braginsky, S. E. Strigin, and S. P. Vyatchanin, Phys. Lett. A 287, 331 (2001).

[19] G. Rempe, R. J. Thompson, H. J. Kimble, and R. Lalezari, Opt. Lett. 17, 363 (1992).

[20] A. N. Cleland and M. L. Roukes, Nature (London) 392, 160 (1998).

[21] J. G. E. Harris, D. D. Awschalom, F. Matsukura, H. Ohno, K. D. Maranowski, and A.C. Gossard, Appl. Phys. Lett. 75, 1140 (1999).

[22] J. G. E. Harris, R. Knobel, K. D. Maranowski, A.C. Gossard, N. Samarth, and D. D. Awschalom, Phys. Rev. Lett. 86, 4644 (2001).

[23] C. J. Hood, H. J. Kimble, and J. Ye, Phys. Rev. A 64, 033804 (2001).

[24] D. Rugar, R. Budakian, H. J. Mamin, and B. W. Chui, Nature (London) 430, 329 (2004).

[25] T. Carmon, H. Rokhsari, L. Yang, T. J. Kippenberg, and K. J. Vahala, Phys. Rev. Lett. 94, 223902 (2005).

[26] T. J. Kippenberg, H. Rokhsari, T. Carmon, A. Scherer, and K. J. Vahala, Phys. Rev. Lett. 95, 033901 (2005).

[27] A. Pai, S. V. Dhurandhar, P. Hello, and J. Y. Vinet, Eur. Phys. J. D 8, 333 (2000).

[28] D.F. Walls and G. J. Milburn, Quantum optics (Springer, New York, 1995).

[29] J. Wilkie, Phys. Rev. E 70, 017701 (2004).

[30] I. Siddiqi et al., Phys. Rev. Lett. 93, 207002 (2004).

[31] I. Siddiqi et al., Phys. Rev. Lett. 94, 027005 (2005).

[32] M. I. Dykman and M. A. Krivoglaz, Zh. Eksp. Teor. Fiz. 77, 60 (1979)[Sov. Phys. JETP 50, 30 (1979)].

[33] J. S. Aldrigde and A. N. Cleland, Phys. Rev. Lett. 94, 156403 (2005). 